Monolithic echo-less photoconductive switches as a high-resolution detector for terahertz time-domain spectroscopy

K. Maussang, J. Palomo, J.-M. Manceau, R. Colombelli, I. Sagnes, L. H. Li, E. H. Linfield, A. G. Davies, J. Mangeney, J. Tignon, and S. S. Dhillon

Citation: Appl. Phys. Lett. 110, 141102 (2017); doi: 10.1063/1.4979536

View online: http://dx.doi.org/10.1063/1.4979536

View Table of Contents: http://aip.scitation.org/toc/apl/110/14

Published by the American Institute of Physics

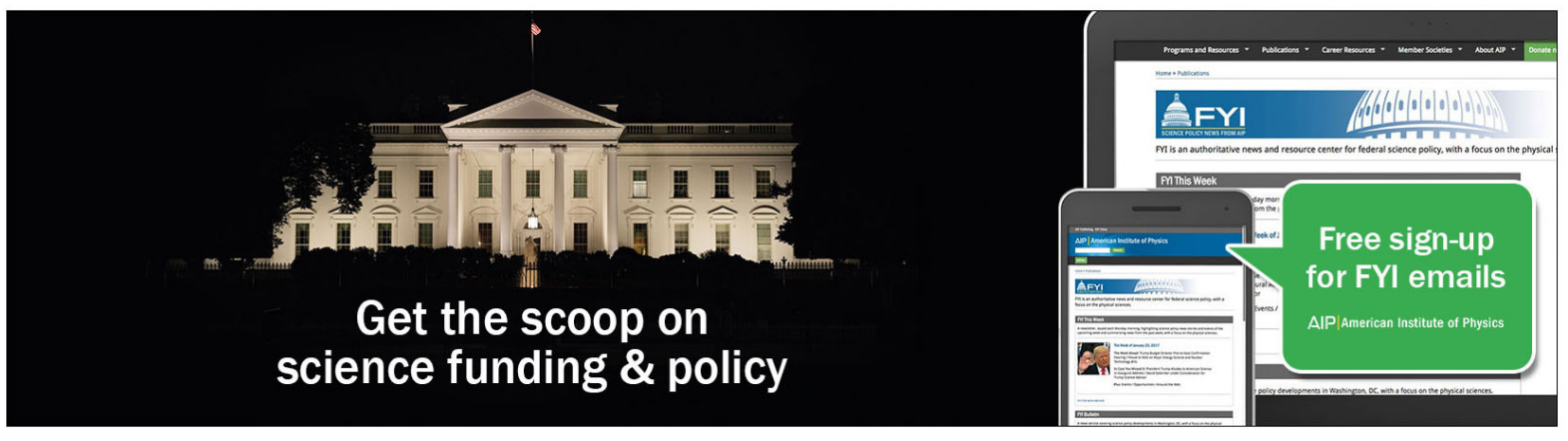




\title{
Monolithic echo-less photoconductive switches as a high-resolution detector for terahertz time-domain spectroscopy
}

\author{
K. Maussang, ${ }^{1, \text { a) }}$ J. Palomo, ${ }^{1}$ J.-M. Manceau, ${ }^{2}$ R. Colombelli, ${ }^{2}$ I. Sagnes, ${ }^{2}$ L. H. Li, ${ }^{4}$ \\ E. H. Linfield, ${ }^{4}$ A. G. Davies, ${ }^{4}$ J. Mangeney, ${ }^{1}$ J. Tignon, ${ }^{1}$ and S. S. Dhillon ${ }^{1}$ \\ ${ }^{1}$ Laboratoire Pierre Aigrain, Ecole Normale Supérieure-PSL Research University, CNRS, Université Pierre \\ et Marie Curie-Sorbonne Universités, Université Denis Diderot-Sorbonne Paris Cité, 24 rue Lhomond, \\ 75231 Paris Cedex 05, France \\ ${ }^{2}$ Centre de Nanosciences et de Nanotechnologies, CNRS, Univ. Paris-Sud, Université Paris-Saclay, \\ C2N-Orsay, 91405 Orsay Cedex, France \\ ${ }^{3}$ Laboratoire de Photonique et de Nanostructures, CNRS (UPR20), Route de Nozay, 91460 Marcoussis, \\ France \\ ${ }^{4}$ School of Electronic and Electrical Engineering, University of Leeds, Leeds LS9 2JT, United Kingdom
}

(Received 6 February 2017; accepted 18 March 2017; published online 3 April 2017)

\begin{abstract}
Interdigitated photoconductive (iPC) switches are powerful and convenient devices for timeresolved spectroscopy, with the ability to operate both as sources and detectors of terahertz (THz) frequency pulses. However, reflection of the emitted or detected radiation within the device substrate itself can lead to echoes that inherently limit the spectroscopic resolution achievable for their use in time-domain spectroscopy (TDS) systems. In this work, we demonstrate a design of lowtemperature-grown-GaAs (LT-GaAs) iPC switches for $\mathrm{THz}$ pulse detection that suppresses such unwanted echoes. This is realized through the growth of a buried multilayer LT-GaAs structure that retains its ultrafast properties, which, after wafer bonding to a metal-coated host substrate, results in an iPC switch with a metal plane buried at a subwavelength depth below the LT-GaAs surface. Using this device as a detector, and coupling it to an echo-less iPC source, enables echofree THz-TDS and high-resolution spectroscopy, with a resolution limited only by the temporal length of the measurement governed by the mechanical delay line used. As a proof-of-principle, the $2_{12}-2_{21}$ and the $1_{01}-2_{12}$ rotational lines of water vapor have been spectrally resolved, demonstrating a spectral resolution below $10 \mathrm{GHz}$. Published by AIP Publishing.
\end{abstract}

[http://dx.doi.org/10.1063/1.4979536]

Advances in terahertz $(\mathrm{THz})$ frequency spectroscopy have resulted in a wide variety of applications from materials science $^{1-3}$ to sensing ${ }^{4,5}$ owing to rapid developments in technologies and systems. With the emergence of ultrafast lasers, a popular technique is $\mathrm{THz}$ time-domain spectroscopy (TDS). This approach provides a broadband spectroscopic capability and overcomes many of the technical difficulties traditionally associated with research in the $\mathrm{THz}$ frequency range, such as the requirement for cryogenic cooling of the sources and detectors. Whilst other spectroscopic methods are conducted directly in the frequency domain, THz-TDS provides direct access to the time-resolved electric field, using femtosecond laser pulses for the generation and detection of single cycle $\mathrm{THz}$ pulses. Although a wide range of methods can be used to generate and detect such $\mathrm{THz}$ pulses, one of the most common methods uses photoconductive switches, ${ }^{6,7}$ which have the advantage of a large spectral bandwidth and a high signal-to-noise ratio. Indeed, most commercial systems exploit such switches for both $\mathrm{THz}$ generation and detection.

In a typical experimental arrangement, metallic contacts are fabricated on top of a semiconductor substrate (such as GaAs) via standard photolithography, and through interband

\footnotetext{
a) Present address: Institut d'Electronique et des Systèmes, CNRS (UMR 5214), Université de Montpellier, 860 rue de Saint-Priest, 34095 Montpellier Cedex 5, France.
}

excitation using an ultrafast laser (e.g., a Ti:Sapphire laser with $\lambda \sim 810 \mathrm{~nm}$ ), photocarriers are generated. When operated as an emitter, the photocarriers are accelerated by a static electric field applied between the electrodes, ${ }^{6,7}$ and the corresponding time-varying current generates an electromagnetic pulse in the $\mathrm{THz}$ frequency range. When operated as a detector, the incident $\mathrm{THz}$ electrical field accelerates the photocarriers, which, when collected by the electrodes, generate a current that is proportional to the $\mathrm{THz}$ electric field. This detection mechanism requires the carrier lifetime to be shorter than the $\mathrm{THz}$ pulse duration (typically $<1 \mathrm{ps}$ ), which is satisfied by using active layers based on low-temperaturegrown GaAs (LT-GaAs). ${ }^{8-10}$

However, for both emitter and detector switches, owing to the finite thickness of the substrate and to the refractive index contrast between the air and the semiconductor, the original pulse is reflected back and forth in the device substrate. This results in the generation of "echoes," i.e., successive $\mathrm{THz}$ pulses separated by the round-trip time between two interfaces, leading to Fabry-Pérot (FP) resonances in the spectrum. For the GaAs based switches used here, this can correspond to a modulation of the spectral amplitude of up to $50 \%$ (Ref. 12) and can greatly affect the possibility to resolve the spectral absorption features. In the case of two closely spaced and weak absorption lines, i.e., weaker modulation than the FP resonances, these are difficult to observe. ${ }^{12}$ In practice, the time-domain scans are truncated to just 
before the first echo, revealing the absorption but limiting the spectral resolution (proportional to the duration of the scan). For strong absorption features, i.e., greater spectral modulation than the FP resonances, although absorptions can be resolved, the clarity of the spectrum is degraded. Consequently, the echoes and hence the FP resonances limit the resolution and precision of THz-TDS. Although the use of silicon hemispherical lenses on the switches can reduce the effect of these echoes, these require a post-processing alignment on the photoconductive switch. ${ }^{11}$ Furthermore, owing to the large area of interdigitated photoconductive (iPC) switches used here, silicon lenses are not adapted as the $\mathrm{THz}$ spot size is large. (The $\mathrm{THz}$ emission from an iPC switch does not act as a point source.) A monolithic scheme for iPC switches is therefore desirable.

In the previous work, we demonstrated an iPC switch geometry for $\mathrm{THz}$ emission that contained a buried metal plane, which eliminated the emission of such echoes. ${ }^{12}$ (Electro-optic sampling using ZnTe crystals was used for the pulse detection.) A metal layer was placed below a $10-\mu \mathrm{m}$ thick GaAs layer, realizing a sub-wavelength $\mathrm{THz}$ bandpass filter. As the $\mathrm{THz}$ generated from the surface comprises wavelengths greater than $70 \mu \mathrm{m}$, this geometry prevents the propagation of the generated $\mathrm{THz}$ pulse into the sample and hence eliminates the echo. The distance between the surface of the emitter and the buried metal plane, $d$, has two critical roles: (i) it determines the spectral band-width in which echo suppression is effective ${ }^{12}$ and (ii) it avoids the bias field distortion from the buried metal plan when the distance $d$ is larger than the electrode separation, ${ }^{12}$ which here is $4 \mu \mathrm{m}$. As mentioned above, to apply this concept to $\mathrm{THz}$ detection, LT-GaAs is required for short carrier lifetimes and low dark currents. However, the use of thick layers of this material is prohibited owing to a reduction in material quality, with layer thicknesses typically limited to $2 \mu \mathrm{m}$. In this work, we develop a sample design and growth methodology that solves this issue, and we demonstrate experimentally that the resulting iPC switch permits echo-less detection in THzTDS systems.

As for the echo-less switch for THz generation, ${ }^{12}$ the device design exploits a wafer bonding process to realise a buried metal layer. To adapt to the use of high quality $2 \mu \mathrm{m}$ thick LT-GaAs for iPC switches, multi-layer growth comprising GaAs and AlGaAs layers were realized to obtain a total switch thickness of $6 \mu \mathrm{m}$. The sample was grown by molecular beam epitaxy (MBE) on a semi-insulating (SI) GaAs wafer. Figure 1(a) shows the layer structure before wafer bonding. From the substrate to the surface, it consists of a 250-nm-thick GaAs buffer layer, a 200-nm-thick $\mathrm{Al}_{0.5} \mathrm{Ga}_{0.5} \mathrm{As}$ etch stop layer, the 2- $\mu \mathrm{m}$-thick $L T$-GaAs active layer, a 300nm-thick $\mathrm{Al}_{0.3} \mathrm{Ga}_{0.7} \mathrm{As}$ electron barrier layer, and a 3.7- $\mu \mathrm{m}$ thick undoped GaAs layer. (A $2 \mu \mathrm{m}$ thick LT-GaAs layer is used to absorb entirely the optical excitation laser pulse.) An electron barrier layer prevents any photoexcited carriers from reaching the GaAs layer. This intermediate sample is annealed at $540{ }^{\circ} \mathrm{C}$ for $15 \mathrm{~min}$ to increase the resistivity. ${ }^{13}$ After annealing, a $300 \mathrm{~nm}$-thick layer of gold was evaporated onto the surface, and the wafer was $\mathrm{Au}-\mathrm{Au}$ bonded onto a host SI-GaAs substrate. The original substrate and the $\mathrm{AlGaAs}$ etch stop were then removed with selective etching (a)

(b)
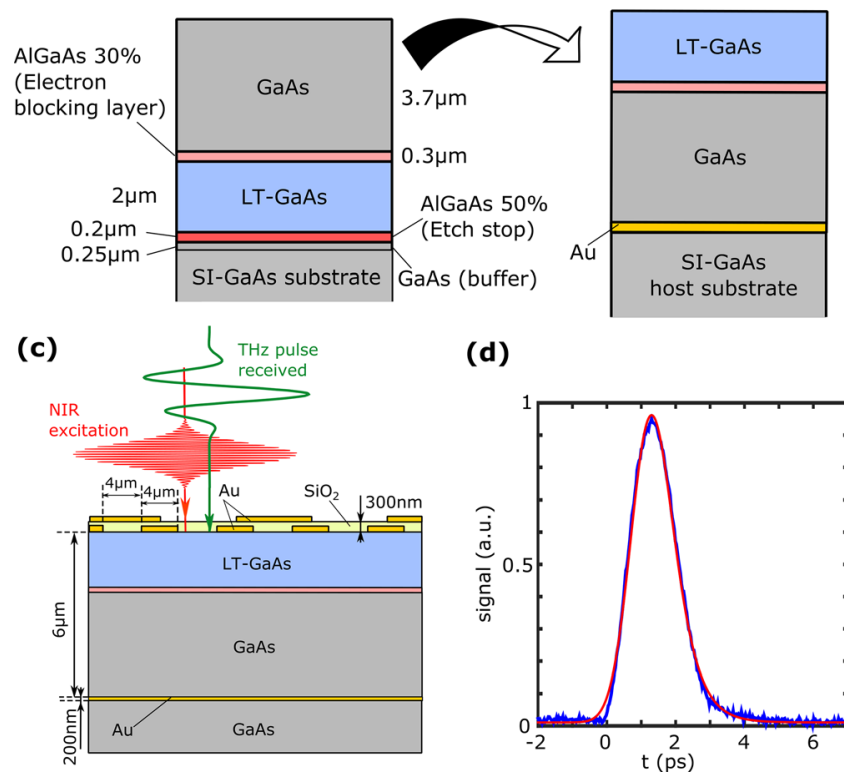

(d)

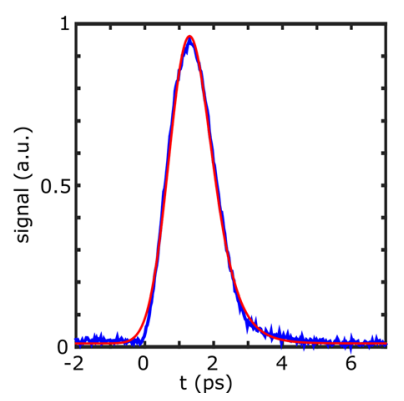

FIG. 1. (a) LT-GaAs growth. The MBE growth sequence on a SI GaAs substrate consists of (i) a 250-nm-thick GaAs buffer, (ii) a 200-nm-thick AlGaAs (50\%) etch stop layer, (iii) $2-\mu \mathrm{m}$-thick LT-GaAs, (iv) $300 \mathrm{~nm}$ of AlGaAs (30\%), and (v) $3.7 \mu \mathrm{m}$ of GaAs. (b) Pre-photolithography sample. The MBE grown sample is wafer bonded to a gold-coated host SI GaAs substrate. The substrate and the AlGaAs (50\%) layer of the MBE grown wafer are removed, exposing the LT-GaAs active region with the echo-blocking metal plane $6 \mu \mathrm{m}$ below the surface. (c) Schematic diagram of the iPC switch, with interdigitated gold electrodes on top of the LT-GaAs layer consisting of $4-\mu \mathrm{m}$-wide electrodes, each separated by $4 \mu \mathrm{m}$. The THz pulse and the femtosecond pulse are focused on the front face of the switch, generating carriers in the LT-GaAs layer with the THz induced current collected from the switch contacts. (d) Experimental measurement of carrier lifetime in the LT-GaAs active layer (blue line). The red line is a numerical fit, showing a lifetime of $\sim 400 \mathrm{fs}$.

using citric and hydrofluoric acid, respectively. This leaves the LT-GaAs active region exposed, with a metal plane of $6 \mu \mathrm{m}$ below the surface, as shown in Figure 1(b). An interdigitated switch geometry ${ }^{14}$ was then fabricated onto the LTGaAs surface, with a design similar to that of Ref. 12, as shown in Figure 1(c). The individual electrodes were $4 \mu \mathrm{m}$ wide, and each was separated by $4 \mu \mathrm{m}$. As for a standard iPC, a second set of metallic fingers with a periodicity double that of the first, and isolated from the interdigitated electrodes by a $300 \mathrm{~nm}$ thick layer of $\mathrm{SiO}_{2}$, was then fabricated. ${ }^{14}$ The total active surface area of the switch was $500 \mu \mathrm{m} \times 500 \mu \mathrm{m}$. An optical pump-THz probe experiment ${ }^{15}$ of the bare LT-GaAs active region showed a carrier lifetime of $\sim 400$ fs (see Figure 1(d)). This confirms that the ultrafast properties of the LTGaAs are preserved despite the buried growth of the layer. ${ }^{13}$

The experimental arrangement used to detect the $\mathrm{THz}$ field is based on the standard $\mathrm{THz}$ time domain spectroscopy. ${ }^{1}$ An iPC emitter switch with a buried metal plane was used to avoid the emission of echoes, ${ }^{12,14}$ biased at $4 \mathrm{~V}$ (corresponding to an applied electric field of $10 \mathrm{kV} / \mathrm{cm}$ ) and excited with an ultrafast (100 fs pulses) Ti:Sapphire oscillator centered at $810 \mathrm{~nm}$. Using parabolic mirrors, the generated $\mathrm{THz}$ pulses were collected from the front surface of the GaAs active layer and focused onto the LT-GaAs detection iPC switch, simultaneously with a probe beam from the femtosecond laser (average power $\sim 10 \mathrm{~mW}$ ). A mechanical 


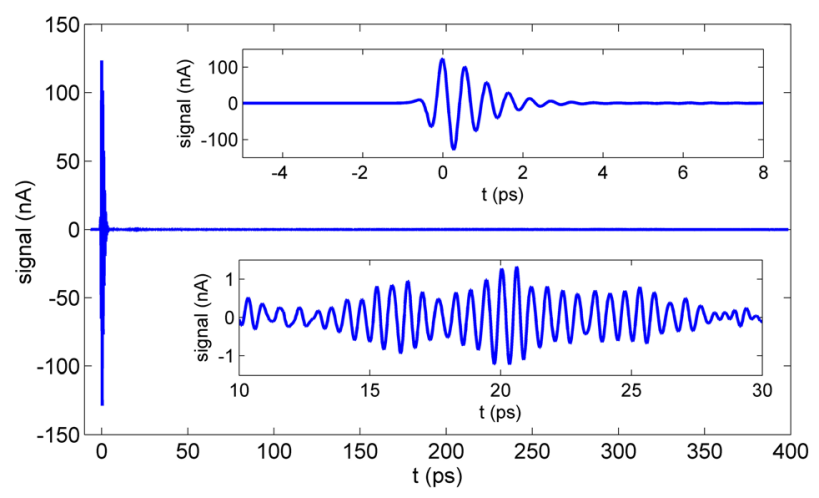

FIG. 2. Detected time-domain trace of the THz electric field with a LTGaAs-based buried metal switch for a scan time of 400 ps. The THz pulse was generated with a $10 \mu \mathrm{m}$ thick GaAs-based buried metal switch. The top inset shows an enhanced view of the THz pulse, and the bottom inset shows a view of the field oscillations owing to residual water absorption.

delay line was used to sample the $\mathrm{THz}$ ultrafast pulse as a function of time. The THz-TDS arrangement is placed in a dry-air purged chamber ( $<2 \%$ humidity) to reduce water absorption. The $\mathrm{THz}$ beam is modulated with a mechanical chopper for lock-in detection at $5 \mathrm{kHz}$.

Figure 2 shows the time-domain trace of the detected $\mathrm{THz}$ field of the echo-less iPC switches. As shown, clearly no echo is visible on the time trace for the total time scan of $400 \mathrm{ps}$, limited by the length of the mechanical delay line. Oscillations visible in the time trace result from residual water vapour in the chamber. From the time trace $E(t)$, the spectral density $\tilde{E}(\omega)$ can be determined by FFT, as shown in Figure 3, with a broad spectral response from about $500 \mathrm{GHz}$ to $3.5 \mathrm{THz}$, limited by the buried metal plane acting as a bandpass filter. ${ }^{12}$ Coherent detection of the electrical field $E(t)$ provides both information on the spectral amplitude $|\tilde{E}(\omega)|$ and the spectral phase $\arg (\tilde{E}(\omega))$. The signal to noise ratio achieved is slightly lower than Ref. 12: this is a consequence of the use of two different detection schemes (electro-optic detection versus photoconductive switch) and lower modulation frequency resulting from the use of a mechanical chopper. Higher modulation frequency should be achievable by electrical modulation of the emitter and careful electromagnetic protection of the detector switch from emitter radiative pick-up noise. In order to illustrate the increase in spectral resolution achieved, one can compare the

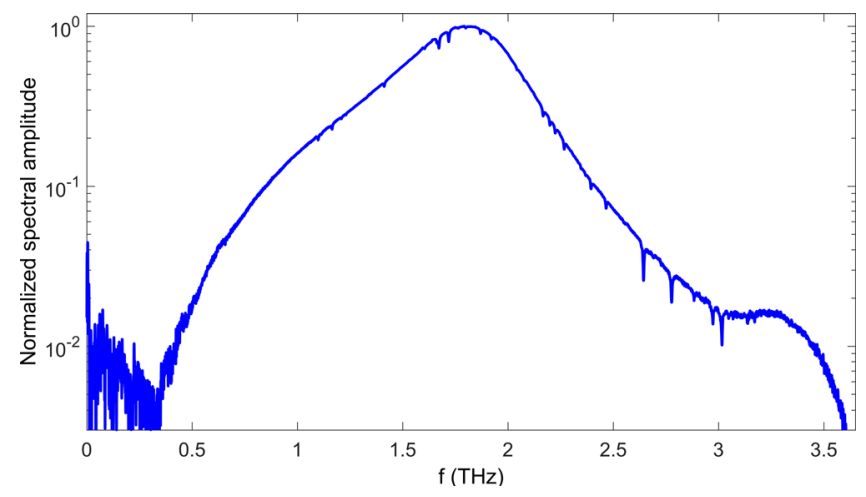

FIG. 3. Spectral amplitude measured by computing the FFT of the time trace of Figure 2. Several absorption features are visible, owing to residual water vapor in the chamber. spectrum measured with different switches both with and without the buried metal plane, with specific absorption lines of residual water vapor in the chamber.

To demonstrate the increase in spectral resolution, we focused on three characteristic rotational transitions of water: $^{16,17} 2_{12}-2_{21}$ at $1.661007 \mathrm{THz}, 1_{01}-2_{12}$ at 1.669904 $\mathrm{THz}$, and $22_{12}-3_{03}$ at $1.716769 \mathrm{THz}$ corresponding to frequency separations of $8.9 \mathrm{GHz}$ and $47 \mathrm{GHz}$, respectively. Measured spectra are shown in Figure 4. In the case of a standard iPC switch as a source (dotted blue curve), the three lines are undistinguishable, merged into a single broad peak as a result of a time window of only 10 ps owing to the first echo from the switch's substrate. ${ }^{13}$ (A $200 \mu \mathrm{m}$ thick $\langle 110\rangle$ ZnTe crystal placed on a $2 \mathrm{~mm}$ thick host substrate was used for electro-optic detection of the electric field. ${ }^{18}$ ) In the case of a buried metal antenna as a $\mathrm{THz}$ source, the time window of $50 \mathrm{ps}$ (limited to the echo from the ZnTe crystal for electro-optic $\mathrm{THz}$ detection ${ }^{18}$ (dashed red curve)) permits the $2_{12}-3_{03}$ line to be resolved from the $2_{12}-2_{21}$ and $1_{01}-2_{12}$ lines. However, the latter two lines are still unresolved. Finally, the use of a buried metal geometry both in emission and detection switches enables one to scan over a time window of $400 \mathrm{ps,}$ resulting in a high spectral resolution (solid black curve) and allowing the $2_{12}-2_{21}$ and $1_{01}-2_{12}$ transitions to be resolved. (Note that with a strong absorption, greater than the amplitude modulation introduced by the FP oscillations, the features can be resolved but with less clarity.) The full-width half maximum of the water lines in the latter case is typically $7 \mathrm{GHz}$. Moreover, since THz-TDS is a coherent detection of the electric field, one has a direct access to the spectral phase. The dispersive profiles of these rotational lines are extracted from the FFT, as shown in Figure 5. The resolution is currently limited by the mechanical delay line but can be extended to times corresponding to the round-trip time of the Ti:Sapphire laser $(76 \mathrm{MHz})$.

We have proposed and realized an echo-less LT-GaAs iPC switch for the coherent detection of $\mathrm{THz}$ radiation in TDS systems. This is based on a multi-layer growth with a buried LT-GaAs, which is wafer bonded to a gold coated host substrate to realize a monolithic echo-less iPC switch. Associated with an echo-less emitter, this provides a

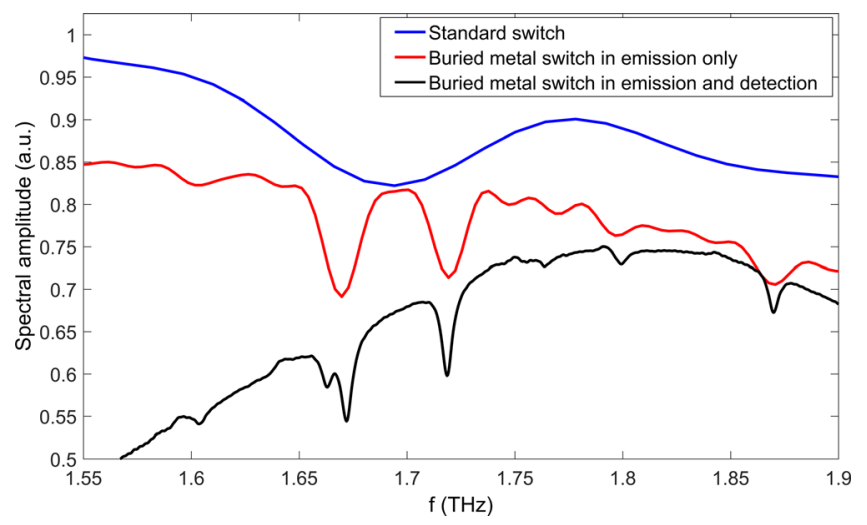

FIG. 4. Comparison of the spectrum obtained with a standard iPC switch as an emitter and a $200 \mu \mathrm{m}$ thick ZnTe crystal for detection (blue curve): a buried metal switch as an emitter but a $200 \mu$ m thick ZnTe crystal for detection (red curve) and a buried metal switch both for emission and detection (black curve). 


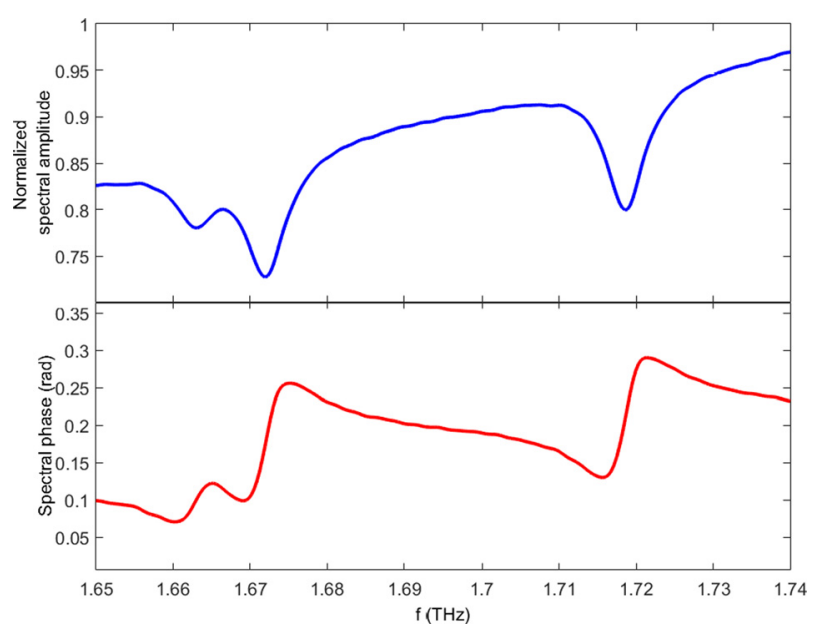

FIG. 5. Enhanced view of the spectral field between $1.65 \mathrm{THz}$ and 1.74 THz. Several absorption lines of water vapour are clearly resolved: $2_{12}-2_{21}$ at $1.661007 \mathrm{THz}, 1_{01}-2_{12}$ at $1.669904 \mathrm{THz}$, and $2_{12}-3_{03}$ at $1.716769 \mathrm{THz}$.

powerful echo-free THz TDS arrangement with high spectral resolution, limited only by the length of the temporal scan achievable. Two rotational lines of water vapour separated by $9 \mathrm{GHz}$ have been experimentally resolved with such an iPC switch. Further work will concentrate on improving the cavity through the design of adapted antenna structures to engineer the spectral and intensity response of the iPC switches. This echo-less THz TDS system would also be relevant for coherent investigations of quantum cascade lasers that require high resolution temporal scans. ${ }^{19-21}$

The authors acknowledge funding from the European Union FET-Open Grant ULTRAQCL 665158. Laboratoire Pierre Aigrain is a "Unité Mixte de Recherche" associated to CNRS (UMR8551), Ecole Normale Supérieure, Université Pierre et Marie Curie (Paris 6), and Université Denis Diderot
(Paris 7). A.G.D. and E.H.L. acknowledge the support from the Royal Society and Wolfson Foundation.

${ }^{1}$ M. Tonouchi, Nat. Photonics 1, 97 (2007).

${ }^{2}$ Y. C. Shen, J. Electr. Electron. Syst. 3, 1 (2003).

${ }^{3}$ M. Beard, G. Turner, and C. Schmuttenmaer, J. Phys. Chem. B 106, 7146 (2002).

${ }^{4}$ D. M. Mittleman, R. H. Jacobsen, R. Neelamani, R. G. Baraniuk, and M. C. Nuss, Appl. Phys. B 67, 379 (1998).

${ }^{5}$ D. Mittleman, Sensing with THz Radiation (Springer, Heidelberg, 2002).

${ }^{6}$ P. U. Jepsen, R. H. Jacobsen, and S. R. Keiding, J. Opt. Soc. Am. B 13, 2424 (1996).

${ }^{7}$ E. Castro-Camus and M. Alfaro, Photonics Res. 4, A36 (2016).

${ }^{8}$ S. Kono, M. Tani, P. Gu, and K. Sakai, Appl. Phys. Lett. 77, 4104 (2000).

${ }^{9}$ Y. C. Shen, P. C. Upadhya, H. E. Beere, E. H. Linflied, A. G. Davies, I. S. Gregory, C. Baker, W. R. Tribe, and M. J. Evans, Appl. Phys. Lett. 85, 164 (2004).

${ }^{10}$ N. T. Yardimci and M. Jarrahi, Sci. Rep. 7, 42667 (2017).

${ }^{11}$ M. C. Nuss and J. Orenstein, "Millimeter and submillimeter wave spectroscopy of solids," Top. Appl. Phys. 74, 7 (1998).

${ }^{12}$ K. Maussang, A. Brewer, J. Palomo, J.-M. Manceau, R. Colombelli, I. Sagnes, J. Mangeney, J. Tignon, and S. S. Dhillon, IEEE Trans. Terahertz Sci. Technol. 6, 20 (2016).

${ }^{13}$ I. S. Gregory, C. Baker, W. R. Tribe, M. J. Evans, H. E. Beere, E. H. Linfield, A. G. Davies, and M. Missous, Appl. Phys. Lett. 83, 4199 (2003).

${ }^{14}$ A. Dreyhaupt, S. Winnerl, T. Dekorsy, and M. Helm, Appl. Phys. Lett. 86, 121114 (2005).

${ }^{15}$ R. Ulbricht, E. Hendry, T. F. Heinz, and M. Bonn, Rev. Mod. Phys. 83, 543 (2011).

${ }^{16}$ X. Xin, H. Altan, A. Saint, D. Matten, and R. R. Alfano, J. Appl. Phys. 100, 094905 (2006).

${ }^{17}$ See http://spec.jpl.nasa.gov. for Values extracted from Nasa database.

${ }^{18}$ Q. Wu and X.-C. Zhang, Appl. Phys. Lett. 67, 3523-3525 (1995).

${ }^{19}$ F. Wang, I. Kundu, L. Chen, L. Li, E. H. Linfield, A. G. Davies, S. Moumdji, R. Colombelli, J. Mangeney, J. Tignon, and S. S. Dhillon, Opt. Express 24, 2174-2182 (2016).

${ }^{20}$ J. Maysonnave, N. Jukam, M. S. M. Ibrahim, R. Rungsawang, K. Maussang, J. Madéo, P. Cavalié, P. Dean, S. P. Khanna, D. P. Steenson, E. H. Linfield, A. G. Davies, S. S. Dhillon, and Jérôme Tignon, Opt. Express 20, 16662 (2012).

${ }^{21}$ D. Oustinov, N. Jukam, R. Rungsawang, J. Madéo, S. Barbieri, P. Filloux, C. Sirtori, X. Marcadet, J. Tignon, and S. Dhillon, Nat. Commun. 1, 69 (2010). 